\title{
ISOMETRIES OF HILBERT SPACE VALUED FUNCTION SPACES
}

\author{
BEATA RANDRIANANTOANINA
}

(Received 30 January 1995; revised 21 April 1995)

Communicated by P. G. Dodds

\begin{abstract}
Let $X$ be a (real or complex) rearrangement-invariant function space on $\Omega$ (where $\Omega=[0,1]$ or $\Omega \subseteq \mathbb{N}$ ) whose norm is not proportional to the $L_{2}$-norm. Let $H$ be a separable Hilbert space. We characterize surjective isometries of $X(H)$. We prove that if $T$ is such an isometry then there exist Borel maps $a: \Omega \rightarrow \mathbb{K}$ and $\sigma: \Omega \rightarrow \Omega$ and a strongly measurable operator map $S$ of $\Omega$ into $\mathscr{B}(H)$ so that for almost all $\omega, S(\omega)$ is a surjective isometry of $H$, and for any $f \in X(H), T f(\omega)=a(\omega) S(\omega)(f(\sigma(\omega)))$ a.e. As a consequence we obtain a new proof of the characterization of surjective isometries in complex rearrangement-invariant function spaces.
\end{abstract}

1991 Mathematics subject classification (Amer. Math. Soc.): 46B04, 46E30.

\section{Introduction}

We study isometries of Hilbert space-valued rearrangement-invariant function spaces $X(H)$, where $\operatorname{dim} H \geq 2$ and $H$ is separable. Our results are valid for both symmetric sequence spaces and non-atomic rearrangement-invariant function spaces on $[0,1]$ with norm not proportional to $L_{2}$ but they are new only in the non-atomic case. If $X$ is a sequence space, not necessarily even symmetric, Theorem 11 is a special case of a much more general result of Rosenthal [14] about isometries of Functional Hilbertian Sums. We include here the case of $X$ being a symmetric sequence space since the proof is essentially the same as when $X$ is a non-atomic rearrangement-invariant function space, and also our techniques are much simpler than those developed in [14].

Spaces of the form $X(H)$ appear naturally in the theory of Banach spaces (see [10, Chapter 2.d]). In particular, if $X$ is rearrangement-invariant (with Boyd indices $\left.1<p_{X} \leq q_{X}<\infty\right)$ then $X\left(L_{2}\right)$ is isomorphic to $X$ ([10, Proposition 2.d.4]) and this plays an important role in the study of the uniqueness of unconditional bases in $X$.

(C) 1996 Australian Mathematical Society 0263-6115/96 \$A2.00+0.00 
Isometries of Hilbert space-valued function spaces have been studied by many authors. In 1974, Cambern [2] characterized isometries of $L_{p}\left(L_{2}\right)$ in the complex case (see also an alternative proof of Fleming and Jamison [5]). Isometries of $L_{p}\left(L_{2}\right)$ in both real and complex cases are described (among other spaces) in the general paper of Greim [7] in 1983. In 1981 Cambern [3] described isometries of both real and complex, $L_{\infty}\left(L_{2}\right)$. In 1986 Jamison and Loomis [8] gave the characterization of isometries in complex Hilbert space-valued non-atomic Orlicz spaces $X\left(L_{2}\right)$. Also there have been a number of studies of various $L_{2}$-valued analytic function spaces. For a fuller discussion of the literature we refer the reader to the forthcoming survey of Fleming and Jamison [4].

We use a method of proof which is designed for spaces over $\mathbb{R}$, but clearly complex linear operators $T: X(H) \rightarrow X(H)$ can be always considered as real linear operators acting on $X(H)\left(\ell_{2}^{2}\right)$ and therefore our results are valid also in the complex case.

Moreover, Theorem 11 with $H=\ell_{2}^{2}$ may be viewed as a statement about the form of isometries of complex rearrangement-invariant spaces. Thus we give a new proof of the fact that all surjective isometries on $X$ can be represented as weighted composition operators, that is, if $T$ is such an isometry, then there are Borel maps $a, \sigma$ such that $T f=a f \circ \sigma$ for all $f$ in $X$ (cf. [17], [18] for non-atomic spaces, and [16] for sequence spaces).

\section{Preliminaries}

We follow standard notations as in [10].

In the following, $H$ denotes a separable Hilbert space with $\operatorname{dim} H \geq 2$. If we want to stress that we restrict our attention to the case when $\operatorname{dim} H=\infty$ we will write $H=\ell_{2}$.

If $X$ is a Köthe function space ([10, Definition 1.b.17]) we denote by $X^{\prime}$ the Köthe $d u a l$ of $X$; thus $X^{\prime}$ is the Köthe space of all $g$ such that $\int|f||g| d \mu<\infty$ for every $f \in X$ equipped with the norm $\|g\|_{X^{\prime}}=\sup _{\|f\|_{X} \leq 1} \int|f \| g| d \mu$. Then $X^{\prime}$ can be regarded as a closed subspace of the dual $X^{*}$ of $X$.

If $X$ is a Köthe function space on $\left(\Omega_{1}, \mu_{1}\right)$ and $H$ is a separable Hilbert space on $\left(\Omega_{2}, \mu_{2}\right)$, we will denote by $\mathbf{X}(\mathbf{H})$ the Köthe function space on $\left(\Omega_{1} \times \Omega_{2}, \mu_{1} \times \mu_{2}\right)$ with the following norm:

$$
\left\|f\left(\omega_{1}, \omega_{2}\right)\right\|_{X(H)}=\|\| f\left(\omega_{1}, \cdot\right)\left\|_{2}\right\|_{X} .
$$

This definition coincides with the notion of $H$-valued Bochner spaces.

It is well-known that $(X(H))^{*}=X^{*}(H)$, and that the space $(X(H))^{\prime} \subset X^{*}(H)$ can be identified with the space of functions $\varphi: \Omega_{1} \rightarrow H$ such that for every $y \in H$ the map $\omega_{1} \longmapsto\left\langle\varphi\left(\omega_{1}\right), y\right\rangle$ is measurable and the map $\varphi_{\#}: \omega_{1} \longmapsto\left\|\varphi\left(\omega_{1}\right)\right\|_{H}$ belongs 
to $X^{\prime}$. The operation of $\varphi$ on $X(H)$ is given by

$$
\varphi(f)=\int_{\Omega_{1}}\left\langle\varphi\left(\omega_{1}\right), f\left(\omega_{1}\right)\right\rangle d \mu_{1}\left(\omega_{1}\right)
$$

for any $f \in X(H)$. Thus $(X(H))^{\prime}=X^{\prime}(H)$.

For any function $f \in X(H)$ we define the map $f_{\#}: \omega_{1} \rightarrow \mathbb{R}$ by $f_{\#}(\omega)=\|f(\omega)\|_{H}$. Then $f_{\#} \in X$. We say that functions $f, g \in X(H)$ are disjoint in a vector sense if $f_{\#}$ and $g_{\#}$ are disjointly supported, that is, $f_{\#}(\omega) \cdot g_{\#}(\omega)=0$ for a.e. $\omega \in \Omega_{1}$. We say that an operator $T: X(H) \rightarrow X(H)$ is disjointness preserving in a vector sense if $(T f)_{\#} \cdot(T g)_{\#}=0$ whenever $f_{\#} \cdot g_{\#}=0$.

We will say that an operator $T: X(H) \rightarrow X(H)$ has a canonical vector form if there exists a non-vanishing Borel function $a$ on $\Omega$ (where $\Omega=[0,1]$ if $X$ is nonatomic or $\Omega \subset \mathbb{N}$ if $X$ is a sequence space) and an invertible Borel map $\sigma: \Omega \rightarrow \Omega$ such that, for any Borel set $B \subset \Omega$, we have $\mu\left(\sigma^{-1} B\right)=0$ if and only if $\mu(B)=0$ and a strongly measurable map $S$ of $\Omega$ into $\mathscr{B}(H)$ (that is, for each $h \in H$ the mapping $\omega \mapsto S(\omega) h$ is measurable) so that $S(t)$ is an isometry of $H$ onto itself for almost all $t$ and $T f(t)=a(t) S(t)(f(\sigma(t)))$ a.e. for any $f \in X(H)$.

Note that the name 'a canonical vector form' is introduced here only for the purpose of this paper - we do not know the standard name for this type of operator. We will need the following simple observation (cf. [9, Lemma 2.4]):

LEMMA 1. Suppose that $T: X(H) \rightarrow X(H)$ is an invertible operator which has a canonical vector form. Then $T^{\prime}: X^{\prime}(H) \rightarrow X^{\prime}(H)$ exists and has a canonical vector form.

PROOF. Operator $T$ has a representation $T f\left(\omega_{1}\right)=a\left(\omega_{1}\right) S\left(\omega_{1}\right)\left(f\left(\sigma\left(\omega_{1}\right)\right)\right)$ where $a, S, \sigma$ satisfy the above conditions for canonical forms and moreover $a$ is nonvanishing and $\sigma$ is an invertible Borel map with $\mu\left(\sigma^{-1} B\right)=0$ if and only if $\mu(B)=0$. Let $v$ be the Radon-Nikodym derivative of the $\sigma$-finite measure $v(B)=\mu\left(\sigma^{-1} B\right)$.

Then for $f \in X(H), g \in X^{\prime}(H)$ we have

$$
\begin{aligned}
g(T f) & =\int_{\Omega_{1}}\left\langle g\left(\omega_{1}\right), T f\left(\omega_{1}\right)\right\rangle d \mu\left(\omega_{1}\right) \\
& =\int_{\Omega_{1}}\left\langle g\left(\omega_{1}\right), a\left(\omega_{1}\right) S\left(\omega_{1}\right)\left(f\left(\sigma\left(\omega_{1}\right)\right)\right)\right\rangle d \mu\left(\omega_{1}\right) \\
& =\int_{\Omega_{1}}\left\langle a\left(\omega_{1}\right)\left(S\left(\omega_{1}\right)\right)^{\prime}\left(g\left(\omega_{1}\right)\right), f\left(\sigma\left(\omega_{1}\right)\right)\right\rangle d \mu\left(\omega_{1}\right) \\
& =\int_{\Omega_{1}}\left\langle a\left(\sigma^{-1}\left(\omega_{1}\right)\right)\left(S\left(\sigma^{-1}\left(\omega_{1}\right)\right)\right)^{\prime}\left(g\left(\sigma^{-1}\left(\omega_{1}\right)\right)\right), f\left(\omega_{1}\right)\right\rangle v\left(\omega_{1}\right) d \mu\left(\omega_{1}\right),
\end{aligned}
$$

since $(S(\omega))^{*}=(S(\omega))^{\prime}$. 
Thus $T^{*} g \in X^{\prime}(H)$ and

$$
T^{\prime} g\left(\omega_{1}\right)=a\left(\sigma^{-1}\left(\omega_{1}\right)\right) v\left(\omega_{1}\right)\left(S\left(\sigma^{-1}\left(\omega_{1}\right)\right)\right)^{\prime} g\left(\sigma^{-1}\left(\omega_{1}\right)\right) \text { a.e. }
$$

Clearly the map $\omega_{1} \mapsto S\left(\sigma^{-1}\left(\omega_{1}\right)\right)^{\prime}$ is strongly measurable and thus $T^{\prime}$ has a canonical vector form.

A rearrangement-invariant function space (ri. space) [10, Definition 2.a.1] is a Köthe function space on $(\Omega, \mu)$ which satisfies the conditions:

(1) $X^{\prime}$ is a norming subspace of $X^{*}$.

(2) If $\tau: \Omega \rightarrow \Omega$ is any measure-preserving invertible Borel automorphism then $f \in X$ if and only if $f \circ \tau \in X$ and $\|f\|_{X}=\|f \circ \tau\|_{X}$.

(3) $\left\|\chi_{B}\right\|_{X}=1$ if $\mu(B)=1$.

Next we will quickly state a definition of Flinn elements. For fuller description and proofs we refer to $[9,12]$.

We say that an element $u$ of a Köthe space $X$ is Flinn if there exists an $f \in X^{*}$ such that $f \neq 0$ and for every $x \in X$ and $x^{*} \in X^{*}$ with $x^{*}(x)=\|x\|_{X} \cdot\left\|x^{*}\right\|_{X^{*}}$ we have $f(x) \cdot x^{*}(u) \geq 0$. We say that $(u, f)$ is a Flinn pair. We denote by $\mathscr{F}(X)$ the set of Flinn elements in $X$. We will need the following facts:

PROPOSITION 2 ([9, Proposition 3.2]). Suppose $U: X \rightarrow Y$ is a surjective isometry. Then $U(\mathscr{F}(X))=\mathscr{F}(Y)$; furthermore if $(u, f)$ is a Flinn pair then $\left(U(u),\left(U^{*}\right)^{-1} f\right)$ is a Flinn pair.

THEOREM 3 (Flinn, [13, Theorem 1.1], [9, Theorem 3.3]). Let $X$ be a Banach space and $\pi$ a contractive projection on $X$ with range $Y$. Suppose $(u, f)$ is a Flinn pair in $X$. Suppose $f \notin Y^{\perp}$. Then $\pi(u) \in \mathscr{F}(Y)$.

THEOREM 4 ([9, Theorem 4.3]). Suppose $\mu$ is non-atomic and suppose $X$ is an order-continuous Köthe function space on $(\Omega, \mu)$. Then $u \in X$ is a Flinn element if and only if there is a non-negative function $w \in L_{0}(\mu)$ with $\operatorname{supp} w=\operatorname{supp} u=B$, so that:

(a) If $x \in X(B)$ then $\|x\|=\left(\int|x|^{2} w d \mu\right)^{1 / 2}$, and

(b) If $v \in X(\Omega \backslash B)$ and $x, y \in X(B)$ satisfy $\|x\|=\|y\|$, then $\|v+x\|=\|v+y\|$.

The last fact about Flinn elements that we will need is a reformulation of Calvert and Fitzpatrick's characterization of $\ell_{p}$-spaces [1]: 
THEOREM 5. Suppose that $X$ is a sequence space with $\operatorname{dim} X=d<\infty, d \geq 3$, and basis $\left\{e_{i}\right\}_{i=1}^{d}$. Suppose that every element $u$ of $X$ with support on at most two coordinates is Flinn in $X$, that is,

$$
\left\{u \in X: u=a_{i} e_{i}+a_{j} e_{j} \text { for some } i, j \leq d, a_{i}, a_{j} \in \mathbb{R}\right\} \subset \mathscr{F}(X) .
$$

Then $X=\ell_{p}^{d}$ for some $1 \leq p \leq \infty$.

ProOF. By [14, Lemma 1.4] $(u, f)$ is a Flinn pair in $X$ if and only if the projection $P$ defined by $P(x)=x-f(x) u$ has norm 1 in $X$. Hence, if $(u, f)$ is a Flinn pair in $X$ then there is a projection of norm 1 onto the hyperplane ker $f \subset X$.

It is also clear from the definition that if $(u, f)$ is a Flinn pair in $X$ then $(f, u)$ is a Flinn pair in $X^{\prime}$. Therefore there exists a projection of norm 1 onto $\operatorname{ker} u \subset X^{\prime}$ for every $u$ with support on at most two coordinates. But then [1, Theorem 1] asserts that if $d \geq 3$ then $X^{\prime}=\ell_{q}^{d}$ for some $1 \leq q \leq \infty$. Thus $X=\ell_{p}^{d}$.

Finally let us introduce the following notation.

Suppose that $X$ is a non-atomic r.i. space on $[0,1]$ and $n$ is a natural number. Let $e_{i}^{n}=\chi_{\left[(i-1) 2^{-n}, i 2^{-n}\right]}$ for $1 \leq i \leq 2^{n}$. Denote $X_{n}=\left[e_{i}^{n}: 1 \leq i \leq 2^{n}\right]$. If $\operatorname{dim} X<\infty$ then, for the uniformity of notation, we will use $X_{n}=X$ for any $n \in \mathbb{N}$. Notice that $X_{n}^{*}$ can be identified naturally with $X_{n}^{\prime}$.

We now need to introduce a technical definition. We will say that an r.i. space $X$ has property $(P)$ if for every $t>0$,

(a) $\left\|\chi_{\left[0, \frac{1}{2}\right]}\right\|_{X}<\left\|\chi_{\left[0, \frac{1}{2}\right]}+t \chi_{\left[\frac{1}{2}, 1\right]}\right\|_{X}$ if $X$ is a non-atomic function space on [0,1]; or (b) $\left\|e_{1}\right\|_{X}<\left\|e_{1}+t e_{2}\right\|_{X}$ if $X$ is a sequence space with basis $\left\{e_{i}\right\}_{i=1}^{\operatorname{dim} X}$.

We say that $X$ has property $\left(P^{\prime}\right)$ if $X^{\prime}$ has property $(P)$.

Notice that, clearly, if $X$ has property $(P)$ (respectively $\left(P^{\prime}\right)$ ) then for every $n \in \mathbb{N}$, $X_{n}$ has property $(P)\left(\right.$ respectively $\left.\left(P^{\prime}\right)\right)$.

LEMMA 6. ([9, Lemma 5.2]) Any ri. space $X$ has at least one of the properties (P) or $\left(P^{\prime}\right)$.

The reason for introducing property $(P)$ is the following fact which will be important for our applications.

If $v \in X_{n}(H)$ then $v=\left(v_{i}\right)_{i=1}^{2^{n}}$, where $v_{i} \in H$ for all $i$ and $v_{i}=\left(v_{i, j}\right)_{j=1}^{\text {dim } H}$. Similarly for $f \in X_{n}^{\prime}(H), f=\left(f_{i}\right)_{i=1}^{2^{n}}$, and $f_{i}=\left(f_{i, j}\right)_{j=1}^{\operatorname{dim} H} \in H$. In this notation we have:

LEMMA 7. Suppose that $X$ has property $\left(P^{\prime}\right)$ and $v \otimes f$ is a Flinn pair in $X_{n}(H)$. If $\left\|v_{1}\right\|_{2}=\left|v_{11}\right|$ then $f_{11} \neq 0$.

PROOF. Assume that $f_{11}=0$. Then, since $v \otimes f \not \equiv 0$ there exist $i>1$ and $j \geq 1$ such that $f_{i j} \neq 0$ and $v_{i j} \neq 0$. In fact $v_{i j} f_{i j}>0$ since $f\left(e_{i j}\right) \cdot e_{i j}^{*}(v) \geq 0$. 
Consider $e_{11}^{*}+t e_{i j}^{*} \in X_{n}^{\prime}\left(\ell_{2}^{d}\right)$. Then

$$
\left\|e_{11}^{*}+t e_{i j}^{*}\right\|_{X_{n}^{\prime}(H)}=\left\|e_{1}^{*}+t e_{i}^{*}\right\|_{X_{n}^{\prime}}>\left\|e_{1}^{*}\right\|
$$

for all $t \neq 0$ since $X$ has property $\left(P^{\prime}\right)$. Hence for any $t \neq 0$, if an element $\left(a_{t} e_{11}+b_{t} e_{i j}\right)$ in $X_{n}(H)$ is norming for $\left(e_{11}^{*}+t e_{i j}^{*}\right)$, then $b_{t} \neq 0$. In fact $b_{t} \cdot t>0$. Let us take $t=-v_{11} /\left(2 v_{i j}\right)$. Then $\operatorname{sgn} b_{t}=\operatorname{sgn} t=-\operatorname{sgn}\left(v_{11} \cdot v_{i j}\right)=-\operatorname{sgn}\left(v_{11} f_{i j}\right)$. Furthermore,

$$
\begin{aligned}
& f\left(a_{t} e_{11}+b_{t} e_{i j}\right) \cdot\left(e_{11}^{*}-\frac{v_{11}}{2 v_{i j}} e_{i j}^{*}\right)(v) \\
& =b_{t} f_{i j} \cdot\left(v_{11}-\frac{v_{11}}{2 v_{i j}} v_{i j}\right)=\frac{1}{2} b_{t} \cdot f_{i j} \cdot v_{11}<0,
\end{aligned}
$$

and the resulting contradiction with numerical positivity of $v \otimes f$ proves the lemma.

\section{Main results}

We start with with an important (for us) proposition about the form of Flinn elements in $X_{n}(H)$. In the case when $\operatorname{dim} H<\infty$ our proof requires a certain technical restriction on the space $X$, which is irrelevant in the case when $H=\ell_{2}$. We present here proofs for both cases since they are quite different. However, for the application to Theorem 11 we need only to know the validity of Proposition 8 .

PROPOSITION 8. Suppose that $X$ is an ri. space with property $\left(P^{\prime}\right), \operatorname{dim} X \geq 3$ and such that the norm of $X$ is not proportional to the $L_{p}$-norm for any $1 \leq p \leq \infty$. Then there exists $N \in \mathbb{N}$, such that if $n \geq N$ and $u=\left(u_{i}\right)_{i=1}^{2^{n}} \in \mathscr{F}\left(X_{n}(H)\right)$, then there exists $1 \leq i_{0} \leq 2^{n}$ such that $\left\|u_{i}\right\|_{2}=0$ for all $i \neq i_{0}$.

REMARK. Proposition 8 can be also understood as a statement about the form of 1-codimensional hyperplanes in $X_{n}(H)$ which are ranges of a norm-1 projection.

PROOF. Let $n$ be big enough so that $X_{n} \neq \ell_{p}^{2^{n}}, 1 \leq p \leq \infty$. Let $u \in \mathscr{F}\left(X_{n}(H)\right)$. Then $u=\left(u_{i}\right)_{i=1}^{2^{n}}, u_{i} \in H$. Let $m=\operatorname{card}\left\{i: u_{i} \not \equiv 0\right\}$. We want to prove that $m=1$.

By Proposition 2 we can assume without loss of generality that $u_{i} \not \equiv 0$ for $i=$ $1, \ldots, m, u_{i}=0$ for $i>m$ and $\alpha_{1}=\left\|u_{1}\right\|_{2}=\min \left\{\left\|u_{i}\right\|_{2}: i=1, \ldots, m\right\}$. Now, for any numbers $\alpha_{2}, \ldots, \alpha_{m} \in \mathbb{R}$ with $\left|\alpha_{1}\right|, \ldots,\left|\alpha_{m}\right| \leq \alpha_{1}$ there exist isometries $\left\{U_{i}\right\}_{i=1}^{m}$ of $H$ such that $\left(U_{i}\left(u_{i}\right)\right)_{1}=\alpha_{i}$ for $i=1, \ldots, m$. Hence by Proposition 2 the element $v$ with

$$
v_{i}= \begin{cases}U_{i}\left(u_{i}\right) & \text { if } i \leq m \\ 0 & \text { if } i>m\end{cases}
$$


is Flinn in $X_{n}(H)$. By Theorem 3 and Lemma $7, \bar{v}=\left(v_{i, 1}\right)_{i=1}^{2^{n}} \in \mathscr{F}\left(X_{n}\right)$. Since the sequence $\left\{\alpha_{2}, \ldots, \alpha_{m}\right\}$ is arbitrary, this implies that every element with support of cardinality less than or equal to $m$ is Flinn in $X_{n}$. But if $m \geq 2$, Theorem 5 implies that $X_{n}=\ell_{p}^{2^{n}}$ for some $1 \leq p \leq \infty$, contrary to our assumption. So $m=1$.

As mentioned above, in the case when $H=\ell_{2}$, Proposition 8 is valid for any r.i. space $X$. Namely we have:

PROPOSITION 9. Let $X_{n}$ be a n-dimensional ri. space not isometric to $\ell_{2}^{n}(n \geq 2)$. If $u=\left(u_{i}\right)_{i=1}^{n} \in \mathscr{F}\left(X_{n}\left(L_{2}\right)\right)$, then there exists $1 \leq i_{0} \leq n$ such that $\left\|u_{i}\right\|_{2}=0$ for all $i \neq i_{0}$.

REMARK. We use here the notation $L_{2}$ for the separable Hilbert space to stress the fact that it is non-atomic. Clearly $L_{2}$ is isometric to $\ell_{2}$ and $X_{n}\left(L_{2}\right)$ is isometric to $X_{n}\left(\ell_{2}\right)$ via a surjective isometry which preserves disjointness in a vector sense and hence our result is valid also in $X_{n}\left(\ell_{2}\right)$.

PROOF. Let $u \in \mathscr{F}\left(X_{n}\left(L_{2}\right)\right)$ be such that $m=\operatorname{card}\left\{i: u_{i} \not \equiv 0\right\}$ is maximal. By Proposition 2 we can assume without loss of generality that $u_{i} \equiv 0$ for $i=m+1, \ldots, n$ and $\operatorname{supp} u_{i}=[0,1]$ for $i=1, \ldots, m$.

If we consider $X_{n}\left(L_{2}\right)$ as a function space on $\{1, \ldots, n\} \times[0,1]$, then $\operatorname{supp} u_{i}=$ $\{1, \ldots, m\} \times[0,1]=B$. Since $X_{n}\left(L_{2}\right)$ is non-atomic, we can apply Theorem 4 to conclude that there exists a measurable function $w$ such that $\operatorname{supp} w=B$ and for every $x \in X_{n}\left(L_{2}\right)(B)$,

$$
\|x\|=\left(\int|x|^{2} w d \mu\right)^{1 / 2} .
$$

Since $X_{n}$ and $L_{2}$ are r.i., $w$ is constant, say $w \equiv k$. We need to show that $m=1$.

Firstly, notice that $m<n$ since $X_{n}$ is not isometric to $\ell_{2}^{n}$ and (1). Assume, to obtain a contradiction, that $m \geq 2$, and consider any element $z=\left(z_{i}\right)_{i=1}^{n} \in X_{n}\left(L_{2}\right)$ such that $z_{i} \equiv 0$ for $i=m+2, \ldots, n$. Define $v, x, y \in X_{n}\left(L_{2}\right)$ by

$$
v_{i}=\left\{\begin{array}{ll}
0 & \text { if } i \neq m+1, \\
z_{m+1} & \text { if } i=m+1
\end{array} ; \quad x_{i}=\left\{\begin{array}{ll}
z_{i} & \text { if } i \leq m, \\
0 & \text { if } i>m
\end{array} ; \quad y_{i}= \begin{cases}\|x\|_{2} & \text { if } i=1, \\
0 & \text { if } i>1\end{cases}\right.\right.
$$

respectively. Then $\operatorname{supp} v \cap B=\emptyset, x, y \in X_{n}\left(L_{2}\right)(B)$ and $\|x\|=\|y\|$, so by Theorem 4(b), $\|v+x\|=\|v+y\|$, that is, $\|z\|=\|v+y\|$. Since $X_{n}$ is r.i.,

$$
\|v+y\|=k\left(\left\|z_{m+1}\right\|_{2}^{2}+\|x\|_{2}^{2}\right)^{1 / 2}=k\|z\|_{2} .
$$

Hence $\|z\|=k\|z\|_{2}$ for every $z \in X_{n}\left(L_{2}\right)(\{1, \ldots, m+1\} \times[0,1])$ and Theorem 4 quickly leads to a contradiction with maximality of $m$. 
PROPOSITION 10. Suppose that $H$ is a separable Hilbert space and $X$ is a rearrangement-invariant function space with norm not proportional to the $L_{2}$-norm. Suppose further that either $X$ is non-atomic on $[0,1]$ or is a sequence space $(\operatorname{dim} X \leq \infty)$, and

(a) $H=\ell_{2} ;$ or

(b) $H=\ell_{2}^{d}, X$ has a norm not proportional to an $L_{p}$-norm for any $1 \leq p \leq \infty, X$ satisfies property $\left(P^{\prime}\right)$ and $\operatorname{dim} X \geq 3$.

Then every surjective isometry $T: X(H) \rightarrow X(H)$ preserves disjointness in a vector sense.

PROOF. We will present the proof in the case when $X$ is non-atomic. If $X$ is a sequence space the proof is almost identical and slightly simpler.

Let us denote $e_{i, j}^{n}=e_{i}^{n} \otimes e_{j} \in X_{n}(H)$ ( $e_{j}$ denotes elements of the natural basis of $H$ ) and $f_{i, j}^{n}=T e_{i, j}^{n}$ for $j, n \in \mathbb{N}, i \leq 2^{n}$.

Define for any $\omega \in[0,1] \times \mathbb{N}($ or $\omega \in[0,1] \times\{1, \ldots, d\}$ in case (b))

$$
F_{n}(\omega)=\sum_{i=1}^{2^{n}} \sum_{j=1}^{\infty} f_{i, j}^{n}(\omega) e_{i, j}^{n} .
$$

Following the same argument as in [9, Theorem 6.1] we see that for almost every $\omega, F_{n}(\omega) \in \mathscr{F}\left(X_{n}^{\prime}(H)\right)$.

For the sake of completness we present this argument here.

Denote by $\Pi(X(H))$ the set of pairs $\left(x, x^{*}\right)$ where $x \in X(H), x^{*} \in X^{\prime}(H)$ and $1=\|x\|=\left\|x^{*}\right\|=x^{*}(x)$.

We note first that by [9, Proposition 2.5], $T^{-1}$ is $\sigma\left(X(H), X^{\prime}(H)\right)$-continuous and so has an adjoint $S=\left(T^{-1}\right)^{\prime}: X^{\prime}(H) \rightarrow X^{\prime}(H)$. We define $g_{i}^{n}=S e_{i}^{n}$. Suppose $\left(x, x^{*}\right) \in \Pi\left(X_{n}(H)\right)$ where $x=\sum a_{i, j} e_{i, j}^{n}$ and $x^{*}=\sum a_{i, j}^{*} e_{i, j}^{n}$. Then $\left(T x, S x^{*}\right) \in$ $\Pi(X(H))$ and this implies that

$$
\left(\sum_{i=1}^{2^{n}} \sum_{j=1}^{\infty} a_{i, j} f_{i, j}^{n}(\omega)\right)\left(\sum_{i=1}^{n} \sum_{j=1}^{\infty} a_{i, j}^{*} g_{i, j}^{n}(\omega)\right) \geq 0
$$

for $\mu$-a.e. $\omega \in \Omega$.

Using the fact that $\Pi\left(X_{n}(H)\right)$ is separable it follows that there is a set $\Omega_{0}^{n}$ of measure zero so that if $\omega \notin \Omega_{0}^{n}$, (2) holds for every $\left(x, x^{*}\right) \in \Pi\left(X_{n}(H)\right)$. Let $\Omega_{0}=\bigcup_{n \geq 1} \Omega_{0}^{n}$.

Now define $G_{n}(\omega)=\sum_{i=1}^{2^{n}} \sum_{j=1}^{\infty} g_{i, j}^{n}(\omega) e_{i, j}^{n} \in X_{n}(H)$. The above remarks show that if $\omega \notin \Omega_{0}$ then $x^{*}\left(G_{n}(\omega)\right) \cdot F_{n}(\omega)(x) \geq 0$ for all $\left(x, x^{*}\right) \in \Pi\left(X_{n}^{\prime}(H)\right)$, that is, $F_{n}(\omega) \in \mathscr{F}\left(X_{n}^{\prime}(H)\right)$ provided that $G_{n}(\omega) \neq 0$ and $\omega \notin \Omega_{0}$. We will show that this happens for a.e. $\omega \in[0,1]$.

Let $B_{n}=\left\{\omega: G_{n}(\omega)=0\right\}$. Clearly $\left(B_{n}\right)$ is a descending sequence of Borel sets. Let $B=\bigcap B_{n}$. If $\mu(B)>0$ then there exists a non-zero $h \in X(H)$ supported on $B$ 
with $\left\langle h, S x^{\prime}\right\rangle=0$ for every $x^{\prime} \in X^{\prime}(H)$. Thus $T^{-1} h=0$, which contradicts the fact that $T$ is an isometry.

Let $D_{n}=\Omega \backslash\left(\Omega_{0} \cup B_{n}\right)$. Then $\mu\left(D_{n}\right)=0$, and if $\omega \in D_{n}$, then $G_{n}(\omega) \neq 0$, and so it follows that $F_{n}(\omega) \in \mathscr{F}\left(X_{n}^{\prime}\right)$. Hence, by Proposition 8 ,

$$
\text { for a.e. } \omega \quad \exists i_{\omega} \quad \text { so that } f_{i, j}^{n}(\omega)=0 \quad \forall i \neq i_{\omega}, j \in \mathbb{N} \text {. }
$$

Let $v_{1}, v_{2}$ be any natural numbers. Consider the isometry $V$ of $H$ defined by

$$
V\left(e_{j}\right)= \begin{cases}e_{j} & \text { if } j \neq v_{1}, v_{2}, \\ \frac{1}{\sqrt{2}}\left(e_{v_{1}}+e_{\nu_{2}}\right) & \text { if } j=v_{1} \\ \frac{1}{\sqrt{2}}\left(e_{v_{1}}-e_{v_{2}}\right) & \text { if } j=v_{2}\end{cases}
$$

and the induced isometry $\bar{V}$ of $X(H)$ defined by $V$ on each fiber.

$$
\bar{V} T e_{i, j}^{n}(t, v)= \begin{cases}f_{i, j}^{n}(t, v) & \text { if } v \neq v_{1}, v_{2}, \\ \frac{1}{\sqrt{2}}\left(f_{i, j}^{n}\left(t, v_{1}\right)+f_{i, j}^{n}\left(t, v_{2}\right)\right) & \text { if } v=v_{1}, \\ \frac{1}{\sqrt{2}}\left(f_{i, j}^{n}\left(t, v_{1}\right)-f_{i, j}^{n}\left(t, v_{2}\right)\right) & \text { if } v=v_{2} .\end{cases}
$$

Similarly as in 3 we conclude that for almost every $t$ there exists $\bar{l}_{\left(t, v_{1}\right)}$ such that $\bar{V} T e_{i, j}^{n}\left(t, v_{1}\right)=0$ for all $i \neq \bar{l}_{\left(t, v_{1}\right)}$. Therefore, for a.e. $t$,

$$
f_{i, j}^{n}\left(t, v_{1}\right)+f_{i, j}^{n}\left(t, v_{2}\right)=0 \quad \forall i \neq \bar{l}_{\left(t, v_{1}\right)}, \forall j
$$

Combining this with (3) we get that for almost every $t \in[0,1]$ and any $v_{1}, v_{2} \epsilon$ $\mathbb{N}, \bar{l}_{\left(t, v_{1}\right)}=i_{t, v_{1}}=i_{t, \nu_{2}}$. It follows easily that $T$ preserves disjointness of functions supported in disjoint dyadic intervals.

We are now ready to present the main result of this paper.

THEOREM 11. Suppose that $X$ is a rearrangement-invariant function space with norm not proportional to the $L_{2}$-norm. Suppose further that either $X$ is non-atomic on $[0,1]$ or it is a sequence space $(\operatorname{dim} X \leq \infty)$, and let $H$ be a separable Hilbert space.

Suppose that $T: X(H) \rightarrow X(H)$ is a surjective isometry. Then there exists a non-vanishing Borel function a on $\Omega$ (where $\Omega=[0,1]$ if $X$ is non-atomic or $\Omega \subset \mathbb{N}$ if $X$ is a sequence space) and an invertible Borel map $\sigma: \Omega \rightarrow \Omega$ such that, for any Borel set $B \subset \Omega$, we have $\mu\left(\sigma^{-1} B\right)=0$ if and only if $\mu(B)=0$, and a strongly measurable map $S$ of $\Omega$ into $\mathscr{B}(H)$ so that $S(t)$ is an isometry of $H$ onto itself for almost all $t$ and

$$
T f(t)=a(t) S(t)(f(\sigma(t))) \text { a.e. }
$$


for any $f \in X(H)$.

Moreover, if $X$ is not equal to $L_{p}[0,1]$, up to equivalent renorming, then $|a|=1$ a.e. and $\sigma$ is measure-preserving.

PROOF. We prove the theorem under the assumption that either:

(a) $H=\ell_{2}$; or

(b) $H=\ell_{2}^{d}, X$ has a norm not proportional to an $L_{p}$-norm for any $1 \leq p \leq \infty, X$ satisfies property $\left(P^{\prime}\right)$ and $\operatorname{dim} X \geq 3$.

If $\operatorname{dim} X=2$ the theorem follows from [14, Theorem 3.12]. If $X=L_{p}[0,1]$, $p \neq 2$ the theorem was proved by Greim [6] and Cambern [3]. If $X$ does not satisfy property $\left(P^{\prime}\right)$ then $X^{\prime}$ does and the result follows by a duality argument. That is, [9, Proposition 2.5] says that the isometry $T$ is $\sigma\left(X, X^{\prime}\right)$ continuous and thus $T$ has an adjoint $T^{\prime}: X^{\prime}(H) \rightarrow X^{\prime}(H)$ which is a surjective isometry. Since $X^{\prime}$ satisfies property $\left(P^{\prime}\right), T^{\prime}$ has a canonical vector form. By Lemma $1, T^{\prime \prime}$ and hence $T$, has a canonical vector form.

So in the following we assume that the assertion of Proposition 10 holds, that is, the isometry $T$ preserves disjointness.

We follow almost exactly the argument of Sourour [15, Theorems 3.1 and 3.2].

Let $\left\{x_{n}\right\}$ be the countable linearly independent subset of $H$ whose linear span $\mathscr{D}$ is dense in $H$ and let $\mathscr{D}_{0}$ be the set of all linear combinations of $\left\{x_{n}\right\}$ with rational coefficients. For any measurable set $E$ let $\Phi(E)=\bigcup_{n} \operatorname{supp}\left(T\left(\chi_{E} x_{n}\right)\right)$. Then, since $T$ is $1-1, \Phi$ is a set-isomorphism.

Let $y_{n}=T\left(\underline{x}_{n}\right)$, where $\underline{x}_{n}$ denotes the function from $X(H)$ constantly equal to $x_{n}$. For every $t \in \Omega$ define $A(t) x_{n}=y_{n}(t)$ and extend $A(t)$ linearly to $\mathscr{D}$; thus for every $y \in \mathscr{D}, A(\cdot) y=T(y)$ a.e.

We will now extend $A(t)$ to a bounded operator on $X$. Let $E \subset \Omega$ be measurable and $y \in \mathscr{D}_{0}$; then

$$
\begin{aligned}
\left\|A(t) y \chi_{\Phi(E)}\right\|_{X(H)} & =\left\|T(\underline{y})(t) \chi_{\Phi(E)}\right\|_{X(H)} \\
& =\left\|T\left(\underline{y} \chi_{E}\right)\right\|_{X(H)}=\left\|\underline{y} \chi_{E}\right\|_{X(H)} \\
& =\left\|\chi_{E}\right\|_{X}\|y\|_{2} .
\end{aligned}
$$

By absolute continuity we can define for almost every $t$ :

$$
a(t)=\lim _{\substack{\mu(E) \rightarrow 0 \\ t \in E}} \frac{\left\|\chi_{\Phi^{-1}(E)}\right\|_{X}}{\left\|\chi_{E}\right\|_{X}}
$$

(notice that if $X=L_{p}$ then $a(\cdot)$ coincides with the function $h(\cdot)$ considered by Sourour).

By (4), $A(t)=a(t) S(t)$ a.e., where $S(t)$ is an isometry of $H$. 
The strong measurability of $S$ and surjectivity of almost all $S(t)$ follow as in the proof of Sourour without change.

Thus, similarly as in [15],

$$
T f(t)=a(t) S(t)(\Phi(f))(t) \text { for a.e. } t \in \Omega,
$$

for some set isomorphism $\Phi$. But, by [11], every set isomorphism of $[0,1]$ is induced by a point isomorphism, that is, there exists an invertible Borel map $\sigma: \Omega \rightarrow \Omega$ such that, for any Borel set $B \subset \Omega$, we have $\mu\left(\sigma^{-1} B\right)=0$ if and only if $\mu(B)=0$ and $(\Phi(f))(t)=f(\sigma(t))$ for a.e. $t \in[0,1]$. Clearly, if $\Omega \subset \mathbb{N}$ then every set isomorphism is a point isomorphism. Thus we have

$$
T f(t)=a(t) S(t)(f(\sigma(t))) \text { a.e. }
$$

for any $f \in X(H)$.

The final remark is now an immediate consequence of [9, Theorem 7.2].

\section{Acknowledgements}

I wish to express my gratitude to Professor Nigel Kalton for his interest in this work and many valuable disscussions.

\section{References}

[1] B. Calvert and S. Fitzpatrick, 'Characterizing $\ell_{p}$ and $c_{0}$ by projections onto hyperplanes', Boll. Un. Math. Ital. 5 (1986), 405-410.

[2] M. Cambern, 'The isometries of $L^{p}(X ; K)$ ', Pacific J. Math. 55 (1974), 9-17.

[3] — 'Isometries of measurable functions', Bull. Austral. Math. Soc. 24 (1981), 13-26.

[4] R. J. Fleming and J. E. Jamison, 'Isometries of Banach spaces - a survey', in: Analysis, geometry and groups: a Riemann legacy (Hadronic Press, Palm Harbor, 1993) pp. 52-123.

[5] —_ 'Classes of operators on vector-valued integration spaces', J. Austral. Math. Soc. 24 (1977), 129-138.

[6] J. Goldstein, 'Groups of isometries on Orlicz spaces', Pacific J. Math. 48 (1973), 387-393.

[7] P. Greim, 'Isometries and $L^{p}$-structure of separably valued Bochner $L^{p}$-spaces', in: Measure theory and its applications. Proc. Conf. Sherbrooke 1982 (eds. J. M. Belley, J. Dubois and P. Morales), Lecture Notes in Math. 1033 (Springer, Berlin, 1983) pp. 209-218.

[8] J. E. Jamison and I. Loomis, 'Isometries of Orlicz spaces of vector valued functions', Math. Z. 73 (1986), 33-39.

[9] N. J. Kalton and B. Randrianantoanina, 'Surjective isometries of rearrangement-invariant spaces', Quart. J. Math. Oxford Ser. (2) 45 (1994), 301-327.

[10] J. Lindenstrauss and L. Tzafriri, Classical Banach spaces, vol. 2: Function spaces (Springer, Berlin, 1979). 
[11] J. von Neumann, 'Einige Sätze über messbare Abbildungen', Ann. of Math. 33 (1932), 574-586.

[12] B. Randrianantoanina, Isometries of function spaces (Ph.D. Thesis, University of MissouriColumbia, 1993).

[13] H. P. Rosenthal, 'Contractively complemented subspaces of Banach spaces with reverse monotone (transfinite) bases', Longhorn Notes, The University of Texas Functional Analysis Seminar (19845), 1-14.

[14] — 'Functional Hilbertian sums', Pacific J. Math 124 (1986), 417-467.

[15] A. R. Sourour, 'The isometries of $L_{p}(\omega, X)$ ', J. Funct. Anal. 30 (1978), 276-285.

[16] K. W. Tam, 'Isometries of certain function spaces', Pacific J. Math. 31 (1969), 233-246.

[17] M. G. Zaidenberg, 'On the isometric classification of symmetric spaces', Soviet Math. Dokl. 18 (1977), 636-640.

[18] - Special representations of isometries of function spaces (in Russian) Studies in the theory of functions of several variables No. 174 (Yaroslavl, 1980) pp. 84-91.

\section{Department of Mathematics}

The University of Texas at Austin

Austin, TX 78712

e-mail: brandri@math.utexas.edu 